\title{
Wells Syndrome as a Rare Cause of Unilateral Ptosis
}

\author{
Caroline Janssen Noémie Lauwers Inge Leysen \\ Department of Ophthalmology, University Hospital of Antwerp, Edegem, Belgium
}

\section{Established Facts}

- Wells syndrome is a rare inflammatory skin disease, with extremities, trunk, and facial region are the most commonly affected area.

\section{Novel Insights}

- Wells syndrome has never been described in a solitary ophthalmological presentation in the absence of skin abnormalities in the face.

\section{Keywords}

Wells syndrome · Eosinophilic cellulitis · Ptosis · Melanoma

\begin{abstract}
We present a case of a woman with a swollen upper eyelid in ptosis, conjunctival nodules, and chemosis present for a couple of weeks. She did not respond to therapy for hordeolum or allergy. A biopsy followed by histopathological examination showed a large infiltration of eosinophilic granulocytes and flame figures, so the diagnosis of Wells syndrome was made. Oral methylprednisolone in high dose was necessary, and tapering was slow because of frequent bouts of relapse. After 3 months, the swelling and hard feeling of the eyelid disappeared, but a slight ptosis remained. Eyelid swelling and eosinophilia recurred 18 months after resolution of the first episode.

(c) 2021 S. Karger AG, Basel
\end{abstract}

\section{Introduction}

Eosinophilic cellulitis, also known as Wells syndrome, is a rare inflammatory skin disease of unknown aetiology [1]. There is no ethnic or gender predisposition and it rarely affects children. Pruritic urticarial erythematous plaques of bacterial cellulitis-like lesions dominate the clinical presentation, with extremities, trunk, and the facial region are the most commonly affected area [2]. Associations with insect bites and nonhaematological malignancy have been seen [3-5]. Facial eosinophilic cellulitis has been described [6]. We report here the first case of a unilateral ptosis caused by Wells syndrome.

$\begin{aligned} & \text { karger@karger.com } \\ & \text { www.karger.com/oop }\end{aligned}$
Karger $\%$

Caroline Janssen

Department of Ophthalmology

University Hospital of Antwerp, Wilrijkstraat 10

BE-2650 Edegem (Belgium)

caroline.janssen2@uza.be 

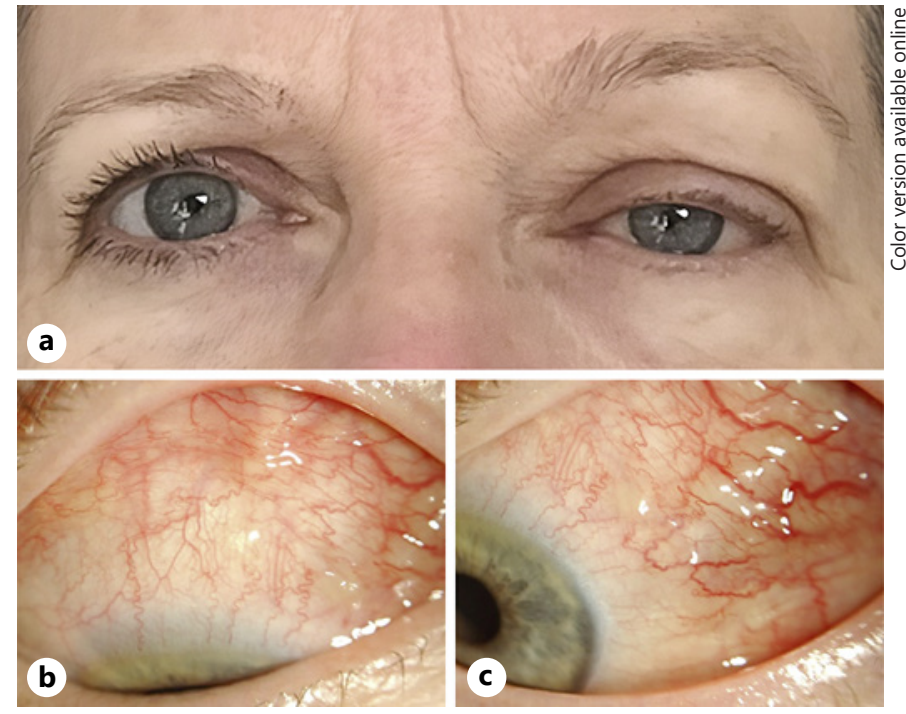

Fig. 1. The woman presented with a swollen left upper eyelid in ptosis (a), bulbar conjunctiva of the left eye with superior (b) and temporal chemosis and subconjunctival nodules (c).

\section{Case Report/Case Presentation}

A 56-year-old woman presented with a 4-week history of a swollen left upper eyelid and ptosis (Fig. 1a) without any systemic symptoms or signs. It had already been treated as a hordeolum with topical tobramycin eye drops for 4 days, then with a combination of tobramycin-dexamethasone drops and ointment for a period of 1 week, followed by oxytetracycline-polymyxin B-hydrocortisone ointment for 10 days without success. The treatment was then stopped. She complained of some slight pain when looking in the superior direction, but there was no diplopia. There was no recalled history of an insect bite. Her medical history included a melanoma in the knee, which was planned for resection. Apart from her eyelid treatments, she was taking no medication. Visual acuity was 20/20 in both eyes, and intra-ocular pressure was normal (RE $19 \mathrm{~mm} \mathrm{Hg}$, LE $21 \mathrm{~mm} \mathrm{Hg}$ ). Slit-lamp biomicroscopy showed a swollen, mild red, and ptotic left upper eyelid with a deeper, palpable hard mass without any evidence of an insect bite. The bulbar conjunctiva had a slight chemosis temporal with a cluster of small subconjunctival nodules which were most prominent superior (Fig. 1b) and temporal (Fig. 1c). There were no signs of intra-ocular inflammation, and fundoscopy was normal. Orthoptic examination showed only a slight mechanical elevation restriction. A blood test was performed in the earliest stage, which showed no inflammatory marker elevation but did show eosinophilia (relative 6.7\%, normal values $0.4-5.0 \%$ and absolute $430 / \mu \mathrm{L}$, normal values $28-273 / \mu \mathrm{L}$ ). Common bacterial, viral, and mycological serology was completely negative, and no autoimmune antibodies were found. An MRI of the orbit showed a soft tissue mass expanding across the levator palpebrae with extensions to the superior and lateral aspects of the eye. After injection of gadolinium contrast, the soft tissue mass enhanced brightly but a distinction between malignancy and an inflammatory/infectious process could not be made. A thorax CT showed no hilar or mediastinal lymphadenopathy and a clear parenchymal lung tissue, so a diagnosis of sarcoidosis was considered unlikely. Ibuprofen $200 \mathrm{mg} 3 \times$ /day was started in combination with esomeprazole $20 \mathrm{mg} /$ day. The lack of clinical response to therapy, 1 week after starting the ibuprofen, led to a biopsy of the conjunctiva and the subcutaneous tissue of the upper eyelid. Dissection of the upper eyelid showed subcutaneous partial fibrotic tissue without a definable mass. Histological examination of the orbital septum, the preaponeurotic fat pad, the musculus orbicularis (Fig. 2a), and the bulbar conjunctiva (Fig. 2b, c) showed infiltration of the tissue by eosinophilic granulocytes. The tissue displayed also a marked histiocytic reaction (Fig. 2b), though there were no signs of vasculitis. Histology of the musculus orbicularis (Fig. 2a) showed fibro-adipose and striated muscle tissue with diffuse infiltration of eosinophils. The eosinophilic infiltrate was also seen between the muscle fibres and the surrounding nerves. Exaggerated flame figures were seen in the granulomatous reaction (Fig. 2b, c) and refer to deposition of eosinophil basic protein on collagen bundles. Given the strong inflammatory infiltrate, infection was high in the differential diagnosis. Gram staining demonstrated blue-purple granules in the tissue, suggestive of gram-positive bacteria, but without neutrophilic infiltration (Fig. 2d), and bacteriological cultures were negative, ultimately leading to the diagnosis of eosinophilic cellulitis. After the initial results of the biopsy, suggesting a gram-positive bacterial infection, topical tobramycin-dexamethasone $4 \times /$ day and oral amoxicillin-clavulanic acid $1 \mathrm{~g}$ $3 \times /$ day was given for 10 days, but this did not result in clinical improvement. One week after the biopsy, the right upper eyelid remained in ptosis and the lesion remained hard on palpation. The conjunctival chemosis slightly improved, but the subconjunctival nodules remained. This failure to respond to therapy led to the commencement of $64 \mathrm{mg}$ of oral methylprednisolone (Medrol) in combination with maintenance of topical tobramycin-dexamethasone $2 \times /$ day. The steroids were then rapidly tapered by reducing the dose in half every 2 days, which led to a slight improvement. The subconjunctival nodules disappeared, but the swollen upper eyelid with the palpable mass and ptosis remained. Blood examinations showed disappearance of eosinophilia $(1.9 \%$, normal values $0.4-5.0,180 / \mu \mathrm{L}$, normal values $28-273 / \mu \mathrm{L}$ ), but as soon as the dose was reduced to 16 mg of methylprednisolone, she noticed the recurrence of the swelling. The methylprednisolone was then increased to $32 \mathrm{mg}$ and was held for 1 week prior to tapering. An oral antihistamine (dihydrochloride $10 \mathrm{mg}$ ) was also added to the treatment. One week later, that is, 7 weeks after the biopsy, the patient was on $16 \mathrm{mg}$ of methylprednisolone, an oral anti-histamine (dihydrochloride $10 \mathrm{mg}$ ), and vitamin $\mathrm{D}$, and she slowly improved. The methylprednisolone was further tapered down by $2 \mathrm{mg}$ every 2 weeks, so that the patient could stop the oral methylprednisolone and the topical tobramycin-dexamethasone after 3 months. The swelling and the hard mass of the right upper eyelid slowly resolved completely, and an MRI of the orbit after 3 months showed no residual mass. A slight ptosis remained at the moment of resolution (1 year after presentation) because of an aponeurotic dehiscence, but surgical correction was not deemed necessary by the patient (Fig. 3a). In the meantime, 7.5 weeks after starting with oral methylprednisolone, the patient was diagnosed with a melanoma (T1a N0 M0, Breslow index 0.72 ) of the knee, which was surgically resected. One year and 6 months after resolution of the first episode, the patient presented with a new swelling of the upper left eyelid. The ptosis also increased but was not covering the pupil (Fig. 3b). A new blood test was performed and showed a recurrence of eosinophilia $(311 / \mu \mathrm{L}$, normal values $28-273 / \mu \mathrm{L})$, but the patient elected to refuse the oral corticosteroid treatment and she remains in follow-up. 
Fig. 2. a Histopathological picture (HE. $\times 50)$ of the musculus orbicularis showing infiltration of the tissue by eosinophilic granulocytes (blue arrow) around the striated muscle (yellow arrow) and the nerves (red arrow). b Histopathological picture $($ HE. $\times 200)$ of the conjunctival epithelium showing infiltration of the tissue by eosinophilic granulocytes and central flame figures (blue arrow). The histiocytes of the granulomatous reaction are also visible (black arrow). c Histopathological picture (HE. $\times 1,000)$ of the conjunctival epithelium showing the eosinophils. d Histopathological picture $(\times 600)$ showing blue-purple granules in the tissue without neutrophilic infiltration.
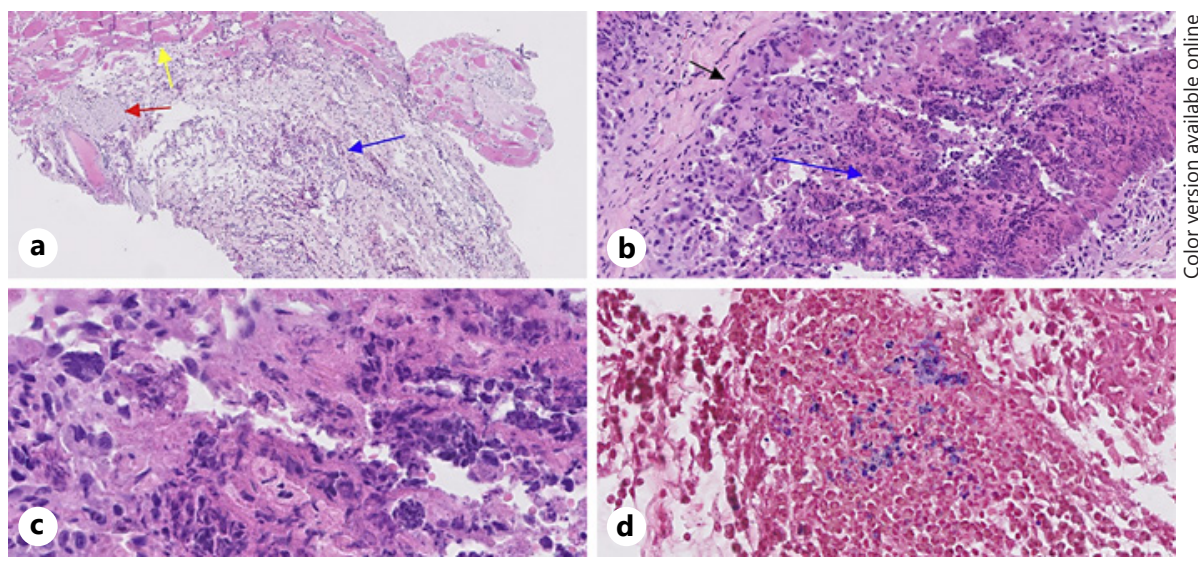

\section{Discussion/Conclusion}

Wells syndrome, also known as eosinophilic cellulitis, is a rare eosinophilic dermatitis first described in 1971 [1]. The disease affects mostly adults, but there is no gender or ethnic predisposition. The pathogenesis is not clear, but a hypersensitivity reaction seems at a prime mechanism [7]. The clinical picture is characterized by infiltrated erythematous plaques, mostly located on the trunk or the limbs, which can cause itching and burning sensation [2]. These lesions can resemble bacterial cellulitis but are resistant to antimicrobial therapy. Peripheral blood eosinophilia, like in our case, is present in half of the patients but is not necessary for the diagnosis. Diagnosis is made on the combination of the clinical findings and typical histopathological findings in biopsy. Histological features can be divided into three groups [8]. First, there is an acute stage with dermal oedema and infiltration of eosinophils in the dermis but no signs of vasculitis. The subacute stage is characterised by eosinophils and flame figures, and the final stage consists of eosinophilic major basic protein and fibrin covering the collagen fibres. Flame figures are not pathognomonic for Wells syndrome, so clinical correlation and exclusion of other causes of flame figures, like eczema and parasitic infection, are always necessary for the diagnosis [9]. During the regression phase, 2-8 weeks after the onset of symptoms, macrophages migrate into the tissue and form granulomatous infiltrates, which can result in resolution of tissue eosinophilia. The interindividual variability of the course of the disease is high. Wells syndrome is known for the relapsing-remitting pattern. Relapses are more frequent in adults and can even occur after a long-term period of remission [10]. The recurrence occurs typically at the same site as the primary episode, like in our case.
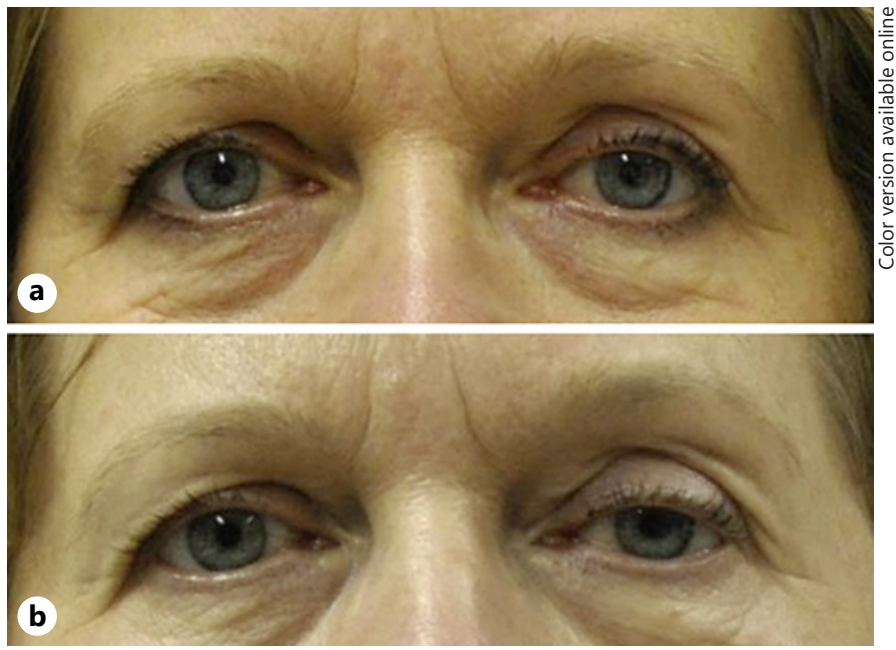

Fig. 3. a Resolution of the swelling and redness of the eyelid. b Recurrence of the swollen left upper eyelid in ptosis 1.5 years after resolution of the first episode.

Wells syndrome has known associations with fungal infections, medications, surgeries, myelofibrosis, and haematological malignancies $[1,11,12]$. Case reports about associations between eosinophilic cellulitis and non-haematological malignancies, like bronchial squamous cell carcinoma, nasopharyngeal carcinoma, colon adenocarcinoma, and renal cell carcinoma, have been described [3-5].

The trigger for eosinophilic cellulitis in our case in not clear. The most obvious trigger would have been a bacterial infection, and the gram-positive structures found in the granulomatous regions are very suggestive, but no neutrophilic reaction was seen. In addition, the antibiotic treatment did not improve the inflammation. An arthropod bite cannot entirely be excluded but the reaction be- 
gan without clear injection point, and it soon involved the bulbar conjunctiva and the levator palpebrae. Furthermore, the biopsy showed only an eosinophilic reaction and no combination of lymphocytes and eosinophils, so it seems not very likely to have been the trigger. The timing of diagnosis of knee melanoma is striking and because of the known association of eosinophilic cellulitis with malignancy we think this also could have been linked.

Despite the fact that spontaneous remission is described, most patients show benefit from pharmacological treatment [13]. Topical and systemic corticosteroid are the most commonly used treatment for Wells syndrome. Recommended starting dose is $1-2 \mathrm{mg} / \mathrm{kg}$ per day. Cortisone can be tapered down over 2-3 weeks. Good results have also been reported with low-dose therapy with $1 \mathrm{mg} / \mathrm{kg}$ prednisolone per day. Other good treatment options that have been described are cyclosporine, dapsone, and antihistamines. Also, the combination of an antihistamine and topical systemic cortisone has shown to be helpful in some case, like in our case.

To our knowledge, solitary ocular Wells syndrome has never been described. This case shows that Wells syndrome can affect the eyelids and the conjunctiva without any other facial signs.

\section{Acknowledgements}

We gratefully thank Dr. Vasiliki Siozopoulu (Department of Histopathology) for the aid with the histopathological description and for making the histopathological pictures. We also gratefully thank Prof. Dr. Sorcha Ni Dhubhghaill for her comments and adaptations of the scientific English spellings and grammar.

\section{Statement of Ethics}

The subject has given her written informed consent to publish her case (including publication of images). All research was conducted ethically in accordance with the Declaration of Helsinki.

\section{Conflict of Interest Statement}

The authors have no conflicts of interest to declare.

\section{Funding Sources}

The authors have no funding to declare.

\section{Author Contributions}

C.J.: design of the work, drafting the work, final approval of the version to be published, and agreement for all aspects of the work in ensuring that questions related to the accuracy or integrity of any part of the work are appropriately investigated and resolved. N.L.: design of the work, critically revising the work, final approval of the version to be published, and agreement for all aspects of the work in ensuring that questions related to the accuracy or integrity of any part of the work are appropriately investigated and resolved. I.L.: design of the work, critically revising the work, final approval of the version to be published, and agreement for all aspects of the work in ensuring that questions related to the accuracy or integrity of any part of the work are appropriately investigated and resolved.

\section{References}

1 Wells GC. Recurrent granulomatous dermatitis with eosinophilia. Trans St Johns Hosp Dermatol Soc. 1971;57(1):46-56.

2 Nielsen T, Schmidt H, Søgaard H. Eosinophilic cellulitis. (Well's syndrome) in a child. Arch Dermatol. 1981;117(7):427-9.

3 Farrar CW, Guerin DM, Wilson NJ. Eosinophilic cellulitis associated with squamous cell carcinoma of the bronchus. Br J Dermatol. 2001;145(4):678-9.

4 Hirsch K, Ludwig RJ, Wolter M, Zollner TM, Hardt K, Kaufmann R, et al. Eosinophilic cellulitis (Wells' syndrome) associated with colon carcinoma. J Dtsch Dermatol Ges. 2005; 3(7):530-1.
5 Rajpara A, Liolios A, Fraga G, Blackmon J. Recurrent paraneoplastic Wells syndrome in a patient with metastatic renal cell cancer. Dermatol Online J. 2014;20(6).

6 Gallard C, Law-Ping-Man S, Darrieux L, Tisseau L, Safa G. Wells syndrome mimicking facial cellulitis: three cases. Ann Dermatol Venereol. 2017;144(4):284-9.

7 Cherng E, McClung AA, Rosenthal HM, Hicks J, Levy ML. Wells' syndrome associated with parvovirus in a 5-year old boy. Pediatr Dermatol. 2012;29(6):762-4.

8 Ladoyanni E, Vlachou C, Thushara R, Snead D. A patient with Wells' syndrome. Clin Exp Dermatol. 2010;35(3):e3-4.

9 Ferreli C, Pinna AL, Atzori L, Aste N. Eosinophilic cellulitis (Well's syndrome): a new case description. J Eur Acad Dermatol Venereol. 1999;13(1):41-5.
10 Caputo R, Marzano AV, Vezzoli P, Lunardon L. Wells syndrome in adults and children: a report of 19 cases. Arch Dermatol. 2006; 142(9):1157-61.

11 Weiss G, Shemer A, Confino Y, Kaplan B, Trau H. Wells' syndrome: report of a case and review of the literature. Int J Dermatol. 2001; 40(2):148-52.

12 Moossavi M, Mehregan DR. Wells' syndrome: a clinical and histopathologic review of seven cases. Int J Dermatol. 2003;42(1):627.

13 Rassler F, Lukacs J, Elsner P. Treatment of eosinophilic cellulitis (Wells syndrome): a systematic review. J Eur Acad Dermatol Venereol. 2016;30(9):1465-79. 